Case Report

\title{
A case report of fungemia due to Kodamea ohmeri
}

\author{
K Prabhu, TR Chinniah \\ Sri Lankan Journal of Infectious Diseases 2021 Vol.11(1):42-46 \\ DOI: http://dx.doi.org/10.4038/sljid.v11i1.8334
}

\begin{abstract}
Kodamea ohmeri is an unusual and emerging fungal pathogen that can cause life threatening infections in humans, particularly in patients with compromised immunity. We report a case of $K$. ohmeri fungemia leading to sepsis that was successfully treated with amphotericin B therapy.
\end{abstract}

Keywords: Kodamea ohmeri, fungemia, amphotericin B

\section{Introduction}

Kodamea ohmeri is an unusual, emerging pathogen of clinical importance. It is a yeast like fungus, frequently misidentified as Candida as they both belong to the same family. ${ }^{1,2}$ Recent studies have shown that this micro-organism can cause life-threatening infections in humans, usually in patients with significant predisposing factors such as diabetes, malignancy, post-chemotherapy neutropenia, immunosuppressive treatments, chronic renal failure and use of indwelling catheters. $^{2,3,4}$ Here, we report a case of $K$. ohmeri fungemia leading to sepsis. To our knowledge this is the first case of $K$. ohmeri sepsis reported from Brunei Darussalam.

\section{Case report}

A 47-year-old man, with known uncontrolled type II diabetes mellitus and epilepsy was admitted to hospital for an abscess on his back. The duration of the abscess was unknown as the patient was unable to give a proper history due to his clinical condition and because he lived alone with no immediate caretaker. On the third day of his admission, incision and drainage of the abscess was done and the patient was transferred to the intensive care unit (ICU) under cefuroxime and gentamicin cover for post-operative management of uncontrolled diabetes with sepsis. Pus from the abscess grew methicillin sensitive Staphylococcus aureus (MSSA) and Streptococcus

Department of Laboratory services. RIPAS Hospital BA1710, Bander Seri Begawan, Brunei Darussalam Address for correspondence: Kavitha Prabhu. Department of Laboratory services. RIPAS Hospital BA1710, Bander Seri Begawan, Brunei Darussalam Telephone +673 8798489 email: kaavitaramesh@yahoo.co.in,

https://orcid.org/0000-0002-7012-8101

Received 30 November 2020 and revised version accepted 30 January 2021

(c) (i) This an open-access article distributed under the terms of the Creative Commons Attribution License, which permits unrestricted use, distribution, and reproduction in any medium, provided the original author and source are credited. 
agalactiae and the blood culture taken on the second day of admission grew MSSA. His antibiotics were changed to cloxacillin and augmentin following the culture results. After six days of ICU management, the patient was transferred to the ward for further management which included investigation of his low hemoglobin level $(6.4 \mathrm{gm} / \mathrm{dl})$ and his learning disabilities, which included difficulties in understanding and following a daily work routine and which led to aggressive behavior.

Two days later, (post admission day 11), the patient developed a swinging fever, partial reduction of movement in his left arm, and upgoing left plantar reflex. The patient's antibiotic therapy was escalated to meropenem. His blood cultures from his peripheral vein and a central venous line taken on the day of fever spike, grew Candida albicans. As a result, the central venous catheter was replaced with a new one, and he was started on fluconazole 200mg OD after the loading dose. Magnetic resonance imaging ruled out spinal pathologies, namely spinal / epidural abscess. CT brain and neck scans showed that he had a right internal jugular artery occlusion, and an infarct in his left parietal lobe. A CT chest showed a cavity in his right lung. One week later (post admission day 17), repeat blood cultures from a peripheral venous cannula and peripheral vein grew multidrug resistant Acinetobacter baumannii. Following this, the peripheral venous catheter was removed and a new one inserted, and amikacin was added to the patient's antibiotic regime.

As the patient continued to have persistent low-grade fever with deranged liver function test results, worsening renal function, and a high $\mathrm{C}$ reactive protein level $(17.68 \mathrm{mg} / \mathrm{dl})$, he was transferred to the ICU on post admission day 32. He was given a stat dose of intravenous clindamycin, started on intravenous colistin, and a new central venous catheter was inserted. Four sets of blood cultures were taken two days apart. Three of the four sets of blood cultures were positive. A yeast was isolated from the BacT/ALERT Microbial Detection System (bioMèrieux SA, Marcy-l'Etoile, France) aerobic blood culture bottles. Subculture showed moist creamy colonies on blood agar and white fluffy colonies on Sabouraud Dextrose Agar (SDA) after 24 hours of incubation at $37{ }^{\circ} \mathrm{C}$. Gram stain of the colony showed yeast cells. The isolate was identified as Kodamea ohmeri by Vitek 2 ID YST system (BioMèrieux SA) and Matrix Assisted Laser Desorption Ionization Time-of-Flight (MALDI-TOF) (Vitek MS, BioMèrieux SA). Antifungal sensitivity was done by Vitek 2 YS07 card (BioMèrieux SA). Minimum Inhibitory Concentration (MIC) for antifungals were as follows: fluconazole $(4 \mu \mathrm{g} / \mathrm{dl})$, voriconazole $(0.12 \mu \mathrm{g} / \mathrm{dl})$, amphotericin B $(0.25 \mu \mathrm{g} / \mathrm{dl})$, caspofungin $(0.25 \mu \mathrm{g} / \mathrm{dl})$, micafungin $(0.12 \mu \mathrm{g} / \mathrm{dl})$, flucytosine $(1 \mu \mathrm{g} / \mathrm{dl})$. The reference method for broth dilution antifungal susceptibility testing of yeasts (CLSI 2012) was used to interpret the antifungal susceptibility results. ${ }^{5}$

The patient's antifungal therapy was changed to liposomal amphotericin B ( $3 \mathrm{mg} / \mathrm{kg} / \mathrm{day})$. There were no vegetations on transesophageal echocardiography. His fever settled after one week of amphotericin B therapy. Blood cultures repeated one week after starting liposomal amphotericin B were sterile. As the patient was clinically stable, he was transferred back to the ward for rehabilitation under neurology after nine days of ICU care. Amphotericin B therapy was given for 14 days. 


\section{Timeline of illness}

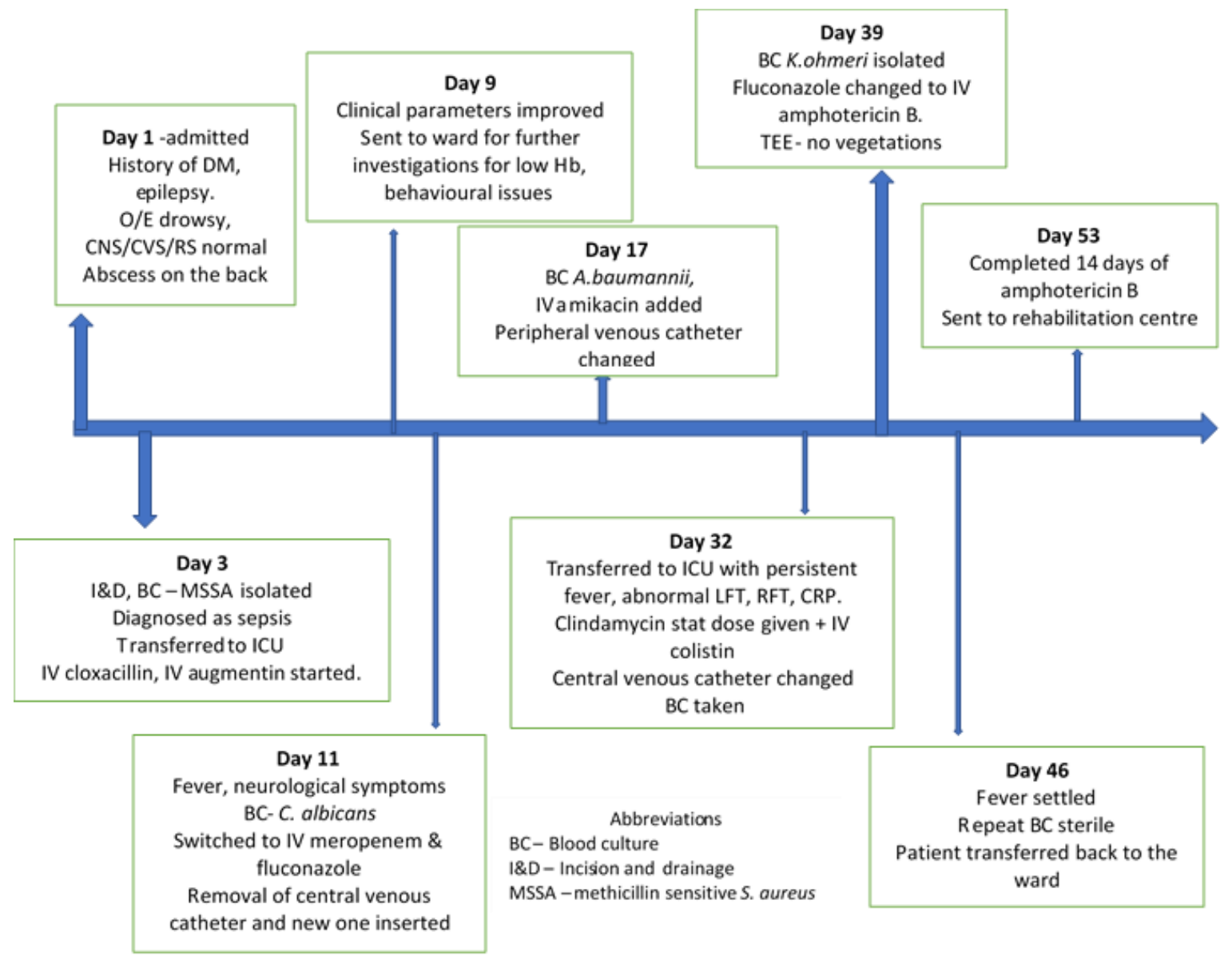

\section{Discussion}

The patient went into sepsis due $K$. ohmeri fungemia. The patient showed signs of recovery after administration of amphotericin B and a change of the central venous catheter.

K. ohmeri, previously known as Pichia ohmeri and Yamadazyma ohmeri, is an asosporogenous yeast, and a telemorph of Candida guillermondii var. membranaefaciens. ${ }^{6}$ The genus Kodamaea has five species and only K. ohmeri shows pathogenicity in humans. ${ }^{6}$

K. ohmeri has been isolated from environmental sources such as sand, pools, sea water and fruits. ${ }^{2}$ The first case of human infection was reported in 1998, and the pathogen, isolated from pleural fluid, was considered a contaminant. ${ }^{7}$ Since then, more infections with this yeast have been reported considering it a true clinical pathogen, especially in patients with underlying immunosuppression. $^{8}$ K. ohmeri can cause fungemia, infective endocarditis, cellulitis, onychomycosis, funguria and peritonitis in neonates and children, immunocompromised adults 
and less frequently in immunocompetent adults. ${ }^{2,3,9}$ Previous antibiotic use, presence of a central venous catheter, parenteral nutrition, chronic renal failure, and cancer were very common among patients. Mortality was high in the case of fungemia but low for other types of infections. ${ }^{2,8,9}$

This patient had diabetes, was on a broad-spectrum of antibiotics, and had a central venous catheter. He could have been infected by $K$. ohmeri through the indwelling central venous catheter, which led to sepsis.

On solid media, K. ohmeri forms Candida like colonies. Although the Vitek 2 ID-YST system is used to correctly identify $K$. ohmeri, some other species of Candida (C. haemoluni) can be mistakenly identified as K. ohmeri. ${ }^{10,11}$ A study by Zhou M et al stated that the Vitek MS system can correctly identify K.ohmeri with $99.9 \%$ confidence. ${ }^{11}$ Molecular diagnosis is the most reliable method for the correct identification of this yeast, but is only available in research laboratories. ${ }^{10}$

In this patient, the organism was identified as $K$. ohmeri by both Vitek 2 ID- YST and Vitek MS system. Antifungal susceptibility done by Vitek 2 showed that this isolate was susceptible-dose dependent (SDD category; CLSI) to fluconazole ${ }^{5}$, susceptible to caspofungin and amphotericin B. Treatment of the patient with amphotericin B had a positive clinical response.

Although there is insufficient data to support a firm treatment recommendation, amphotericin B appears to be an attractive first-line agent and echinocandins are possible alternative candidates. Susceptibility testing is recommended not only to guide treatment but also to provide MICoutcome relationships and hence data for future optimized treatment recommendations. ${ }^{12}$

\section{Conclusion}

K. ohmeri is an emerging human pathogen with high mortality and resistance to fluconazole, which is a commonly used antifungal agent in clinical practice. Therefore, accurate identification of $K$. ohmeri isolates and anti-fungal susceptibility tests to detect MIC values in the clinical microbiology laboratory are vital.

Conflict of interest: None

Ethics statement \& consent for publication: Verbal consent was taken from the caretaker (patient's sister)

Funding source: None

Author contribution: Both authors contributed to writing the manuscript. Acknowledgement: None

\section{References}

1. Chakrabarti A, Rudramurthy SM, Kale P, et al. Epidemiological study of a large cluster of fungaemia cases due to Kodamaea ohmeri in an Indian tertiary care centre. Clin Microbiol Infect. 2014; 20:83-9. doi: 10.1111/1469-0691.12337 
2. Ioannou P, Papakitsou I, Kodamea ohmeri infections in humans: systematic review. Mycoses 2020; 63:636-43. doi: doi.org/10.1111/myc.13094

3. Yang BH, Peng MY, Hou SJ, et al. Fluconazole resistant Kodamaea ohmeri fungemia associated with cellulitis: Case report and review of the literature. International journal of Infectious diseases 2009; 13:e493-7. doi: 10.1016/j.ijid.2009.02.003

4. Shang ST, Lin JS, Ho SJ, et al. The emerging life threatening opportunistic fungal pathogen Kodamaea ohmeri: optimal treatment and literature review. J. Microbiol. Immunol. Infect. 2010; 43:200-6.

doi: 10.1016/S1684-1182(10)60032-1

5. Clinical and Laboratory Standards Institute (CLSI). Reference method for broth dilution antifungal susceptibility testing of yeasts, $4^{\text {th }}$ supplement. Approved standard CLSI document M27-S4. Wayne, PA: Clinical and Laboratory Standards Institute; 2012.

6. Yamada Y, Suzuki T, Matsuda M, et al. The phylogeny of Yamadazyma ohmeri (Etchells et Bell) Billon-Grand based on the partial sequences of $18 \mathrm{~S}$ and $26 \mathrm{~S}$ ribosomal RNAs: the proposal of Kodamaea gen. nov. (Saccharomycetaceae). Biosci Biotechnol Biochem 1995; 59:1172-4.

doi: $10.1271 / b b b .59 .1172$

7. Bergman MM, Gagnon D, Doern GV. Pichia ohmeri fungemia. Diagn Microbiol Infect Dis.,1998; 30:229-231. doi: 10.1016/s0732-8893(97)00233-2

8. Rosalba V, Claudia B, Maria IM, et al. Fungemia due to Kodamaea ohmeri in a young infant and review of the literature. Medical Mycology Case reports. 2016; 13:5-8. doi: 10.1016/j.mmcr.2016.06.001

9. Otag F, Kuvuca N, Erturan Z, et al. An outbreak of $P$. ohmeri infection in the paediatric intensive care unit: case reports and review of the literature. Mycoses 2005; 48:265-9.

doi: 10.1111/j.1439-0507.2005. 01126.x

10. Lee JS, Shin JH, Kim MN et al. Kodamaea ohmeri isolates from patients in a university hospital: Identification, antifungal susceptibility, and Pulsed Field Gel Electrophoresis Analysis. Journal of clinical microbiology 2007; 45:1005-10. doi: 10.1128/JCM.02264-06

11. Zhou M, Yu S, Kudinha T, et al. Identification and antifungal susceptibility profiles of Kodamaea ohmeri based on a seven-year multicenter surveillance study. Infection and drug resistance 2019; 12;1657-64. doi: 10.2147/IDR.S211033

12. Arendrup MC, Boekhout T,Akova M, et al. ESCMID and ECMM joint clinical guidelines for the diagnosis and management of rare invasive yeast infections. Clin Microbial Infect 2014; 20:76-98.

doi: 10.1111/1469-0691.12360 